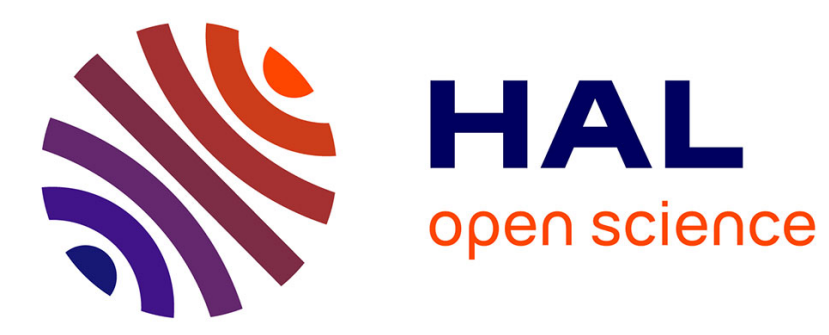

\title{
Corps impérial, corps souffrant au Japon : l'empereur Meiji et la médecine occidentale
}

\author{
Eric Seizelet
}

\section{To cite this version:}

Eric Seizelet. Corps impérial, corps souffrant au Japon : l'empereur Meiji et la médecine occidentale. Revue d'Histoire Moderne et Contemporaine, 2019, 66 (27-51). halshs-02103044

\section{HAL Id: halshs-02103044 https://shs.hal.science/halshs-02103044}

Submitted on 18 Apr 2019

HAL is a multi-disciplinary open access archive for the deposit and dissemination of scientific research documents, whether they are published or not. The documents may come from teaching and research institutions in France or abroad, or from public or private research centers.
L'archive ouverte pluridisciplinaire HAL, est destinée au dépôt et à la diffusion de documents scientifiques de niveau recherche, publiés ou non, émanant des établissements d'enseignement et de recherche français ou étrangers, des laboratoires publics ou privés. 


\section{Corps impérial, corps souffrant au Japon : l'empereur Meiji et la médecine occidentale}

\section{ERIC SEIZELET}

\section{Professeur émérite, Université Paris-Diderot}

Entre 1868 et 1912, le long règne de quarante-cinq ans de l'empereur Meiji (1852-1912) fut pour le Japon une période de profonds changements pendant laquelle l'archipel va accéder à la modernité et asseoir sa position dominante en Asie Orientale. Mais cette mutation n'a pas été exempte d'oppositions ni d'interrogations. L'accession à la modernité passait-elle nécessairement par l'occidentalisation de l'archipel ? L'importation et l'appropriation massive des techniques, des institutions et des idées occidentales n'allaient-elles pas porter un coup fatal à l'identité japonaise ? Fallait-il, au nom du «progrès » et de la « civilisation » jeter aux oubliettes de l'histoire, les pratiques, usages et coutumes «pernicieux du passé » quand bien même certains avaient fait leurs preuves et étaient solidement ancrés dans la mentalité collective ? ${ }^{1}$ Là comme ailleurs, l'introduction progressive de la médecine occidentale n'a pas échappé à ce questionnement.

C'est à partir de l'époque de Meiji que l'élévation du niveau sanitaire de la population devint un enjeu national de santé publique. La « gestion du corps » n'était plus seulement une affaire individuelle, mais collective, et l'introduction, puis la diffusion de la médecine occidentale, fut indissociable de la mise en place de politiques d'hygiène et de santé publique, au nom d'une « amélioration de la race japonaise », dans lesquelles le darwinisme social le disputait aux préoccupations de défense nationale ${ }^{2}$. Par ailleurs, toutes les innovations introduites entre 1868 et 1912 l'ont été au nom de la volonté impériale : Meiji est passé à la postérité comme un monarque éclairé guidant en l'espace d'une génération la Nation sur la voie d'une modernité et d'une prospérité maîtrisées. Mais ce discours ne saurait occulter une réalité : le monarque n'a pratiquement jamais été à l'initiative de ces réformes. Et s'il les a indiscutablement accompagnées durant son règne, le peu que l'on sait de la

1. Pour une réflexion globale sur la modernité japonaise, Pierre-François SoUYRI, Moderne sans être occidental. Aux origines du Japon d'aujourd'hui, Paris, Editions Gallimard, 2016.

2. Bernard Thomann, La naissance de l'Etat social japonais, Paris, Presses de Sciences Po, 2015, p. 96 et s.; également, Philippe CHEMOUILLI, « Les épidémies de choléra et la mise en place d'un système d'hygiène moderne », Cipango, 2004, n¹1. p. 173-208. L'auteur évoque en particulier l'épidémie de choléra de 1886 qui fit 108405 victimes. En 1872, selon des statistiques officielles jugées «optimistes » par les démographes japonais, l'espérance de vie aurait été alors de 42,8 ans pour les hommes et de 44,3 ans pour les femmes. La tuberculose pulmonaire vient très largement en tête des décès par maladie avec 76589 cas en 1908, suivie de la dysenterie avec 8053 cas : Nihon teikoku shiin tôkei, Statistiques des causes de décès dans l'Empire du Japon, Tôkyô, Naikaku tôkei kyoku, 1911-1912, p. 3. La tuberculose pulmonaire représente 7,77\% des décès enregistrés en 1910. Le béribéri tue encore annuellement à la fin de l'époque de Meiji, de 1900 à 1909, entre 6500 et 15085 personnes. 
personnalité de l'empereur Meiji laisse plutôt entrevoir une forte inclination au conservatisme, notamment sur les plans politiques et éducatifs. On crédite ainsi l'empereur Meiji d'avoir modernisé les infrastructures médicales et le système de formation des médecins. Certes, les preuves abondent de la sollicitude impériale pour la santé de la population, notamment des plus démunis. Mais il s'en faut de beaucoup qu'il ait été convaincu de s'appliquer à lui-même les nouveaux protocoles et les nouvelles thérapies issus de la médecine occidentale. Le présent article se propose en conséquence d'expliquer les raisons de ce hiatus entre la volonté impériale « officielle » et la volonté personnelle du tennô - l'empereur en japonais - et ses conséquences sur la nature multiple d'un « corps impérial » qui, en tout état de cause, le distingue, en tant que divinité incarnée, arahito-gami, de la communauté des mortels, sous ses deux espèces : son «corps public »-celui donné à voir - et son «corps naturel » inaccessible, sauf à une poignée de familiers ${ }^{3}$.

L'histoire de la médecine japonaise et de sa rencontre avec l'Occident, dès avant la période de Meiji, les processus d'hybridation et d'acculturation ont donné lieu à une abondante littérature tant en langue japonaise qu'en langues occidentales ${ }^{4}$. La médecine palatiale moderne post-restauration, quant à elle, reste peu documentée, et essentiellement en langue japonaise : annales impériales et annuaires officiels, notes journalières ou réminiscences - souvent tardives - de courtisans, correspondance de médecins impériaux, instructions spécifiques du ministère de la Maison impériale dont il est fait état dans cette étude.

3. La théorie des deux corps du roi d'Ernst Kantorowicz a séduit une partie de l'historiographie japonaise : Takashi FuJITANI, Power and Pageantry in Modern Japan, University of California Press, 1998 p. 155 et s. Cependant, la nature du corps impérial comme « corps précieux » incite à nuancer, comme nous le verrons, cette distinction.

4. Y compris en langue française. Sur les interactions entre la médecine traditionnelle et la médecine occidentale à partir du XVIII ${ }^{\mathrm{e}}$ siècle, les travaux de Mieko MACE font autorité. Voir en particulier Médecins et médecine dans l'histoire du Japon, Paris, Les Belles-Lettres, 2013 ; également Anne MILLERAND, « La modernisation de la médecine japonaise d'Edo à Meiji : rupture ou continuité ? » Thèse pour le doctorat de médecine, Université Pierre et Marie Curie, Paris VI, 4 mai 2011. Sur le versant militaire du développement de la médecine occidentale dans l'archipel, Ken DAIMARU, «Préserver la santé des armées dans le Japon moderne : la médecine militaire face à la guerre russo-japonaise », Thèse de doctorat en histoire contemporaine, Université de Nanterre, Paris X, 16 décembre 2017. En langue anglaise, John C. Berry, « Medicine in Japan: Its Development and Present Status », The Journal of Race Development, avril 1912, vol. 2, n4, p. 455-479. Emiko OHNUKI-TIERNEY, Illness and Culture in Contemporary Japan: An Anthropological View, Cambridge University Press, 1984 ; Shizu SAKAI et alii, Transaction in Medecine \& Heteronomous Modernization: Germany, Japan, Korea and Taiwan, Tokyo, The University of Tokyo Center of Philosophy (UTCP), 2009 ; Hoi-eun KIM, Doctors of Empire: Medical and Cultural Encounters Between Imperial Germany and Meiji Japan, Toronto, Buffalo, London, University of Toronto Press, 2014. En allemand, le journal tenu par Erwin von Bälz reste incontournable : Das Leben eines deutschen Arztes im erwachenden Japan ; Tagebücher, Briefe, Berichte, publié en 1931 à Stuttgart (Engelhorn). 


\section{L'OCCIDENTALISATION DE LA MEDECINE DE COUR A L'EPOQUE DE MEIJI}

$\mathrm{Au}$ lendemain de la Restauration, le gouvernement impérial se lance dans une entreprise de longue haleine et tous azimuts de réforme des institutions. Le Palais n'est pas épargné mais la modernisation des structures médicales du Palais va se heurter à des résistances, tandis que les missions des médecins impériaux, au départ mal définies, vont progressivement se diversifier.

\section{L'évolution des structures médicales du Palais}

Lorsque Meiji monte sur le trône en février 1867, l'infrastructure médicale au Palais n'a guère changé depuis l'Etat régi par les codes du VIII ${ }^{\mathrm{e}}$ siècle de notre ère ${ }^{5}$. Avant 1868, lorsqu'un membre de la famille impériale est malade, l'Office des Soins et Traitements, Ten.i ryô, fait appel à des intendants médicaux officiant sous astreinte ou par roulement au Palais, choisis parmi les familles héréditairement habilitées à fournir les soins et les médicaments à la Cour. Au cours de la seconde moitié de la période d'Edo, l'Office n'est plus seulement ouvert aux familles aristocratiques, mais aux médecins de ville extérieurs, ce qui posa la question de l'intégration des intendants médicaux de l'Office dans la pyramide des titres de Cour, du contrôle du Bakufu sur les nominations et promotions, et notamment de l'autorisation, à titre héréditaire ou non, du port du sabre comme signe de distinction. Les soins alors dispensés combinaient à la fois la «médecine japonaise » et la «médecine chinoise », avec ses connotations taoïstes et bouddhistes. Ils intégraient alors pratiques thérapeutiques, magiques et divinatoires ${ }^{6}$. Plus généralement, il n'existait pas encore de système centralisé de formation des médecins : l'accès à la profession était libre et l'on se formait soit sous la houlette d'un maître, soit dans les écoles spécialisées mises place par les fiefs à partir du XVIII ${ }^{\mathrm{e}}$ siècle, sur la base principalement des classiques médicaux tels que le Shanghan Lun (Traité sur les lésions du froid) rédigés sous la Chine des Han orientaux. Très vite cependant, le nouveau gouvernement issu de la Restauration se trouve confronté à un double problème : quelle place accorder sur le plan national à la médecine occidentale et faut-il introduire cette médecine au Palais ? Cette réflexion a été menée par Takashina Tsunenori (1834-1889), un spécialiste de médecine sino-japonaise, dont l'arrière-grand-père Tôitsu, le grand-père Tsunenobu (1773-1843) le père Tsuneyoshi avaient eux-mêmes servi comme médecins les empereurs Kôkaku (1771-1840) Nintoku (1800-1846) et Kômei (1831-1867). Au cours du $2^{\mathrm{e}}$ mois de 1868 , il co-signe avec son père un mémoire à destination de l'empereur dans lequel il recommande que des occidentalistes comme des

5. Codes d'administration publique et pénale inspirés de la Chine des T'ang au cours de la première expérience japonaise de centralisation. Sur l'histoire des services médicaux du Palais avant 1868, YAMADA Shigemasa, Ten.i no rekishi, Histoire des médecins du Palais, Kyôto, Shibunkaku shuppan, 1980.

6. MiYAKE Muneo, «Meiji tennô go osanai toki no go kenkô ni tsuite », A propos de la santé de l'empereur Meiji, jeune, Nihon ishi gakkai kansai shibu, 1954, n4, p. 1118-1122. 
traditionalistes puissent être nommés comme médecins impériaux, jii, appelle plus largement au développement de la médecine et d'infrastructures médicales occidentales dans l'archipel et à la fin du système de recrutement héréditaire des médecins impériaux ${ }^{7}$. Et c'est grâce à lui qu'un occidentaliste, Ikeda Kensai (1841-1918) - l'un des pères fondateurs de la médecine japonaise moderne et le premier à recevoir le titre de « docteur en médecine » en 1888 - fut autorisé à examiner l'empereur le 22 mai 1870. L'objectif était double : d'une part combler le retard accumulé à la Cour par rapport aux institutions shôgunales qui avaient été plus réceptives, dans les dernières années du régime, à la médecine dite «hollandaise » que la Cour impériale de Kyôto, sous le règne de l'empereur Kômei - un fervent partisan du maintien de la fermeture de l'archipel - avait banni de la capitale ${ }^{8}$. D'autre part, par la combinaison des deux types de médecine, mieux soigner la Famille impériale. Il ne s'agissait donc pas, à l'origine du moins, d'encourager l'abandon de la médecine traditionnelle en faveur de la médecine occidentale. Toutefois, le changement rapide de dénomination des services du palais en charge de la santé de la Famille impériale entre 1868 et 1871 atteste les luttes d'influence que se livrent en coulisses occidentalistes et traditionalistes dans l'entourage du tennô. Mais dès la réforme du ministère des Affaires Suprêmes de septembre 1871, ces luttes d'influence tournent à l'avantage des occidentalistes : les traditionalistes sont soit peu à peu évincés, soit rétrogradés dans la hiérarchie administrative - ils passent de la qualité de médecin à celle auxiliaire médical, iin -, mutés ou quittent le ministère de la Maison impériale pour rentrer à Kyôto. L'entourage médical du jeune empereur Meiji comporte alors dix médecins dont huit sont des adeptes de la médecine occidentale et seule la confiance du souverain permet en fait à Takashina de rester durablement à son poste en tant que médecin impérial. Cette évolution est d'autant plus remarquable qu'en 1874, à l'échelon national, le nombre de traditionalistes autorisés à exercer l'emporte encore largement sur les occidentalistes - 23015 contre 5274 - pour une population globale estimée, selon les nouveaux services de l'état civil, à 33749932 âmes, soit un médecin pour 1192 habitants. A partir de 1888, tous les médecins impériaux sont des occidentalistes ${ }^{9}$. Entre 1868 et 1912, la direction des services

7. On désignera sous l'appellation « occidentalistes » les partisans de la médecine occidentale. Sur le mémorandum précité, accepté par le ministère des Affaires Suprêmes le 30 mars 1868, Sugaya Akira, «Meiji shoki wagakuni no iryô seido no kakuritsu ni kôken shita jinmyaku - Iwasaki Jun, Sagara Tomoyasu, Nagayo Sensai no jiseki wo tsûshin ni shite », Les réseaux qui ont contribué à la consolidation des institutions médicales nationales au début de Meiji. Autour des réalisations d'Iwasaki Jun, Sagara Tomoyasu et Nagayo Sensai, Kôshû eisei, septembre 1974, vol. 38, n 9, p. 464-469.

8. Les autorités shôgunales, à la veille de la conclusion des traités avec les puissances occidentales, avaient définitivement levé les entraves à la «médecine hollandaise » le 11 août 1858.

9. Chiffres établis d'après Naimushô eiseikyoku-hen, Direction de l'hygiène du ministère de l'Intérieur, Dai ichiji dai ni hôkoku, rapports $\mathrm{n}^{\circ} 1$ et 2 , juillet 1875 -juin 1876 . Sur ces différents développements, ENDO Shôji, «Meiji-ki no jii seido to ikeda monjo », L'institution des médecins attachés à la Cour impériale et le fonds Ikeda, in YoshIDA Tadashi et FUKASE Yasuaki (éd.), Higashi to nishi no iryô bunka, La culture médicale de l'Est et de l'Ouest, Kyôto, Shibunkyaku shuppan, 2001, p. 265-306. Le dernier médecin impérial spécialiste de médecine sino-japonaise fut Asada Sôhaku 
médicaux du Palais fut assurée par sept hauts fonctionnaires qui présentent trois caractéristiques communes : ils ont tous étudié la médecine occidentale, soit à Nagasaki, le port d'attache de la médecine dite hollandaise, ou plus tard, à la faculté de médecine de l'Université de Tôkyô ; ils ont complété leur formation à l'étranger, soit en Hollande, soit en Allemagne ; ils ont exercé des fonctions d'enseignement et/ou de direction dans les établissements hospitaliers nouvellement créés. Sur le plan administratif, le directeur des services médicaux du Palais est de rang chokunin, c'est-à-dire qu'il est nommé par l'empereur sur proposition du chef du gouvernement - ministre des Affaires Suprêmes puis Premier ministre après 1885 - après délibération du Cabinet, tandis que les médecins sont soit de rang chokunin, soit de rang sônin, nommés par le chef du gouvernement ou le ministre de la Maison impériale sur sanction impériale. En ce sens, la nomination des directeurs des services médicaux du Palais est alignée sur celle des directeurs des autres services du ministère de la Maison impériale. C'est donc un poste de rang élevé, mais qui ne figure pas dans la liste des emplois du ministère à la discrétion impériale - ceux de rang shinnin dont la nomination procède directement du souverain lors d'une cérémonie spéciale d'investiture et est contresignée par le chef du gouvernement, tels que les Grands chambellans, les Premiers aides-de-camp ou les Gardiens du Sceau Privé ${ }^{10}$.

Par ailleurs, à partir d'août 1874, le ministère de l'Education, sur autorisation du ministère des Affaires Suprêmes, prit un certain nombre d'instructions à destination de préfectures de Tôkyô, Kyôto et Ôsaka visant à la limitation de la qualification de médecins aux seuls praticiens formés à la médecine occidentale. Ce fut le point de départ d'une série de réglementations visant à exclure de fait, puis de droit, les praticiens de la médecine sino-japonaise du corps médical ${ }^{11}$. Il faudra néanmoins attendre la réorganisation politico-administrative postérieure à l'introduction du

(1815-1894), anciennement attaché au $13^{\mathrm{e}}$ shôgun Tokugawa Iesada (1824-1858), qui avait la charge du traitement des enfants impériaux. Il soigna également le ministre de France Léon Roches (1809-1901) et fit partie de l'équipe médicale qui sauva le prince impérial Yoshihito - le futur empereur Taishô - en 1879, lorsqu'il fut atteint de méningite. En septembre 1883, il avait présenté sa démission à la suite du décès consécutifs de deux des filles de l'empereur, mais sa démission avait été refusée. Egalement HoshiKaWA Mitsumasa, « Nihon no jii no rekishi », Histoire des médecins impériaux du Japon, Tôdai daiichi geka dôsokai dayori, 1983, vol. 16, n¹, p. 4 et s ; Margaret LoCK, "The organization and practice of East Asian medicine in Japan: continuity and change». Social Science Medicine. Part B: Medical Anthropology, 1980, 14(4), p. 245-253.

10. Shokuinroku Meiji 22 nen, Répertoire de la fonction publique centrale, année 1889, Tôkyô, Insatsukyoku, p. 23-24, Kokuritsu kokkai toshokan dijitaru korekushon, Bibliothèque nationale de la Diète collections numériques (désormais KKTDK), http://dl.ndl.go.jp/info:ndljp/pid/779759. Site consulté le 13 avril 2018. La majorité des directeurs sont issus du corps des médecins impériaux.

11. Proclamation du ministère des Affaires Suprêmes $\mathrm{n}^{\circ} 35$ du 23 octobre 1883, portant réglementation de la certification de médecin, Ishi menkyo kisoku, KKTDK http://dl.ndl.go.jp/info:ndljp/pid/2943301 ; ordonnance du ministère des Affaires Suprêmes n³4 du même jour portant réglementation de l'examen préalable à l'ouverture de la profession médicale, Ijutsu kaigyô shiken kisoku, ibidem. Site consulté le 6 avril 2018. 
régime constitutionnel en 1889 pour que, sous l'impulsion d'Ikeda Kensai, le palais se dote de médecins impériaux, jii, permanents issus de la Faculté de médecine de l'Université de Tôkyô, et formés à la médecine occidentale. Et en 1895, la Diète repousse - par 105 voix contre 78 - la tentative des traditionalistes d'obtenir eux aussi leur qualification en tant que médecin. Un autre élément de réflexion fut de mener des enquêtes sur l'organisation de la médecine royale ou impériale dans les cours étrangères ${ }^{12}$. En somme si, primitivement, la qualité de médecin impérial, jii, n'est pas liée à la détention d'un diplôme de médecine occidentale, la conjonction de la réforme des conditions d'accès à la profession médicale avec les réorganisations successives des services spécialisés du ministère de la Maison impériale aboutit au monopole de l'Université de Tôkyô dans le recrutement des médecins impériaux. Ce départ des traditionalistes du Palais s'explique cependant moins par l'infériorité supposée de leur art que parce qu'ils avaient battu la bataille du pouvoir contre les occidentalistes. Néanmoins, il s'en fallait cependant de beaucoup que la médecine traditionnelle soit éradiquée dans le pays. En effet, la prédominance de la médecine occidentale dans les instances du pouvoir et la formation des élites implique des changements de paradigme anthropologiques et symboliques qui vont au-delà de la simple substitution ou réception de techniques médicales exogènes, et qui n'obéissent pas au même rythme. Surtout, la médecine traditionnelle, exclue de toute reconnaissance officielle, n'a pas déserté les habitudes, pratiques médicales et médicinales en vigueur dans la population. De plus, la nouvelle réglementation n'empêchait pas - en théorie du moins - les traditionalistes d'exercer leur métier pourvu qu'ils fussent pourvus d'un diplôme de médecine occidentale. Enfin, bien que devenus minoritaires au Palais, les adeptes de la médecine sino-japonaise continuèrent, malgré tout, à jouer un rôle non négligeable dans l'entourage de l'empereur qui ne s'est résolu qu'à contrecœur à leur marginalisation progressive au Palais.

\section{Rôle et missions des médecins impériaux}

Dans les premières années de Meiji, la composition, les fonctions et le nombre des agents en charge de la médecine impériale resteront floues. Plus tard, la direction des affaires médicales du ministère de la Maison impériale se bornera à indiquer, laconiquement, qu'elle à la « charge des questions relatives aux examens médicaux et à l'hygiène du Palais ». En d'autres termes les compétences de cet organe sont conçues en termes généraux ; elles ne sont pas réservées au traitement des seuls membres de la Famille impériale, mais de toutes les questions sanitaires pouvant

12. Ministère de la Maison impériale, ministère des Affaires Etrangères, « Doitsu, Roshia, Itarî, Ôsutoria kaku teikoku teishitsu jii kansei narabi ni teiin sono kinmu gaiki torishirabekata kunaidaijin yori irai no ken », Instruction du ministre de la Maison impériale 50-4 relative aux recherches concernant les régulations, missions, effectifs, ainsi que l'organisation des médecins des maisons impériales d'Allemagne, de Russie, d'Italie et d'Autriche, Japan Center for Asian Historical Records. National Archives of Japan (désormais JACAR, https://www.jacar.archives.go.jp/aj/meta/MetSearch.cgi, référence B15100966100. Consulté le 2 avril 2018. 
affecter les agents travaillant dans l'entourage impérial ${ }^{13}$. Très tôt, l'idée d'une présence médicale stable, sinon permanente, aux côtés de l'empereur s'est imposée pour faire face aux risques sanitaires générés par la nouvelle configuration de la monarchie et l'ouverture de l'archipel : le ministère de la Maison impériale impose la présence de médecins lors de tous les déplacements impériaux impliquant un éloignement de la capitale : revues des troupes, grandes manœuvres, tournées provinciales, déplacement du ministériat des Affaires Suprêmes lors de la guerre civile du sud-ouest en 1877, ou du Grand quartier général impérial (GQGI) à Hiroshima lors de la guerre sino-japonaise (1894-1895). La direction des affaires médicales du ministère est également mobilisée pour dépêcher des médecins impériaux en région en cas de catastrophes naturelles, de tremblements de terre ou sur les théâtres d'opérations. Parfois, il arrive que ce soit l'empereur lui-même qui mandate ses propres médecins : le 16 septembre 1878, en visite dans la région de Niigata où sévit le trachome à l'état endémique, il s'étonne du nombre de personnes atteintes de maladies oculaires. Il ordonne à ses médecins de diligenter une enquête sur les causes de ce phénomène et fait un don de 1000 yens pour les soigner. L'objectif est double : d'une part rendre compte au trône de l'état sanitaire des populations sinistrées et des troupes déployées sur le terrain. D'autre part soutenir le moral des soldats et des populations en témoignant par ce geste de la sollicitude impériale. Il lui arrive également d'envoyer les médecins impériaux, plus rarement son médecin en chef, auprès de hautes personnalités gouvernementales ou de la Cour, malades ou hospitalisées. En outre, le ministère de la Maison impériale, sur avis de la direction des affaires médicales, prend des dispositions spéciales pour limiter, interdire l'accès au Palais et aux appartements privés de l'empereur des agents et fonctionnaires porteurs de germes, malades ou pris de vomissements ${ }^{14}$. C'est également aux médecins médicaux qu'il appartient de contrôler et de goûter les repas servis à l'empereur et qui ne peuvent être préparés que par le service de la Table du Palais. En somme, les médecins impériaux ont des missions générales de soins, d'information et de veille sanitaire.

En 1902, le ministère de la Maison impériale recruta un médecin allemand,

13. Statuts du ministère de la Maison impériale du 4 février 1886, JACAR, https://www.digital.archives.go.jp/das/image/M0000000000001715506, référence $\mathrm{n}^{\circ} \mathrm{A} 15111079300$. Site consulté le 2 avril 2018. La réforme des statuts du ministère intervenue le 23 juillet 1889 précisera que le nombre de médecins et fixé à seize puis à vingt-cinq en 1907 (effectifs budgétaires).

14. «Daihon.ei wo Hiroshima he susumareru kunaishô kokuji », Proclamation du ministère de la Maison impériale relatif au déplacement du GQGI à Hiroshima», ibid., https://www.jacar.archives.go.jp/aj/meta/MetSearch.cgi, référence n A1506007160. Site consulté le 2 avril 2018 ; «Tôkyô-fu ka infuruenza ryûkô no kizashi ni tomonau haietsu-tô ni kansuru jiikyokuchô gushin no ken tsûchi », Avis du ministre de la Maison impériale, le vicomte Tanaka Mitsuaki, relatif aux explications du directeur de la direction des affaires médicales concernant l'épidémie de grippe sévissant dans la préfecture urbaine de Tôkyô, 5 avril 1898, ibid., https://www.jacar.archives.go.jp/aj/meta/image, référence ${ }^{\circ} \mathrm{C} 10061991400$. Site consulté le 2 avril 2018. 
Erwin von Bälz (1849-1913) ${ }^{15}$. En poste au Japon depuis 1876, comme conseiller médical pendant près de trois décennies, il apporta une contribution décisive à la modernisation du système médical et à la formation des médecins japonais. Bälz avait été présenté au Palais par un médecin militaire bien introduit à la Cour, Hashimoto Tsunatsune (1845-1909), et Iwasa Jun (1835-1912), médecin impérial occidentaliste, qui lui avaient confié des conférences auprès de la direction précitée des affaires médicales, et c'est sur leur entremise que le médecin allemand fut amené à soigner les membres de la Famille impériale dont la mère de Meiji - la dame d'honneur Nakayama Yoshiko (1836-1907) - l'impératrice douairière Eishô (1835-1897) en novembre 1893 et le prince héritier. Il fit partie de l'équipe médicale qui, en mars 1899, avec Hashimoto Tsunatsune, Oka Genkei (1852-1925), chef de la direction des affaires médicales recommanda que la princesse Sachiko (1885-1966), fille aînée du prince impérial Fushimi Sadanaru (1858-1923), chez laquelle il avait été décelé un souffle suspect dans la poitrine, ne soit pas mariée au prince héritier Yoshihito comme initialement prévu. Tous les témoignages s'accordent à dire que le Palais et l'empereur tenaient Bälz en haute estime ${ }^{16}$. Il fut en tout cas le seul médecin occidental auquel les autorités japonaises firent appel de façon récurrente au chevet de la Famille impériale, et en particulier auprès du prince héritier, à partir de mars 1902. Un point qui mérite d'être d'autant plus souligné que Meiji se méfiait, comme on le verra, de la médecine occidentale et que la présence d'un praticien étranger au Palais pouvait susciter des réserves ${ }^{17}$. Il faut souligner cependant que Bälz n'a jamais eu l'occasion de soigner l'empereur et qu'au moins jusqu'en 1888, en dépit de la prédominance des occidentalistes au Palais, les enfants impériaux continuèrent à être soignés par les traditionalistes ${ }^{18}$.

«CORPS PRECIEUX », CORPS PUBLIC ET CORPS POLITIQUE DE L'EMPEREUR

15. Il y a une ambiguïté sur le statut exact du docteur Bälz au Palais, certaines sources japonaises indiquent qu'il fut médecin impérial, d'autres consultant médical auprès du ministère de la Maison impériale, Kunaishô goyôgakari, qui n'est pas un poste de titulaire.

16. ISHIBASHI Chôei et OGAWA Teizô, Oyatoi gaikokujin 9 igaku, Les étrangers au service du gouvernement japonais, Tôkyô, Kashima kenkyûjo shuppankai, 1969, tome 9, La médecine, p. 125.

17. Dans une lettre en date du 9 février 1878, Fukuzawa Yukichi (1835-1901), l'un des intellectuels éclairés les plus en vue du Japon de Meiji, fait l'éloge d'Obata Einosuke (1850-1909), un dentiste formé à la médecine occidentale qu'il recommande au ministère de la Maison impériale en tant chef médecin responsable de la santé dentaire du monarque. Obata était à l'époque le seul médecin de Tôkyô officiellement qualifié en tant que dentiste par le ministère de l'Intérieur. Outre l'excellence de sa formation et sa réputation, Fukuzawa invoque un argument de prestige : la nomination d'un étranger constituerait un camouflet pour l'intéressé et l'ensemble de la profession dentaire japonaise, Keiô gijuku enshû, Fukuzawa Yukichi shokan zenshû, Intégrale de la correspondance de Fukuzawa Yukichi, Tôkyô, Iwanami shoten, 2001, vol. 2, p. 53. Cette suggestion ne fut pas acceptée et il n'existe pas de traces indiquant que l'empereur ait subi des soins bucco-dentaires, en dépit de sa mauvaise dentition. En revanche, l'un de ses confrères occidentalistes, Takayama Kisai (1851-1933), prit en charge à partir de 1887 la santé dentaire du prince héritier et de l'impératrice.

18. Asukai Masamichi, Meiji daitei, Meiji le Grand, Tôkyô, Bungei shunjû, 2017, p. 240. 
La Restauration de 1868 va apporter au corps impérial des changements importants, dans le souci de se démarquer de l'ancien régime du bakufu et pour manifester aux Puissances les signes extérieurs et symboliques des mutations en cours. Le corps public de l'empereur sera donc mis en conformité avec les impératifs de la modernisation. Dans le même temps, en tant que « corps précieux », le corps impérial restera soumis à des contraintes de sanctification qui lui confèrent un statut particulier.

\section{La modernisation du corps public de l'empereur}

Les initiatives gouvernementales vont progressivement « naturaliser » le corps public du tennô en le dépouillant des attributs vestimentaires, des artifices de maquillage et de composition visagiste qui singularisaient le statut du tennô avant la Restauration. D'abord dans son physique : le ministère des Affaires Suprêmes avait en effet autorisé, le 23 septembre 1871, les Japonais à ne plus nouer leurs cheveux par le chignon traditionnel. Et c'est le 20 mars 1873 que l'empereur fit couper le sien, adopta définitivement la coiffure occidentale, renonça au fard, à la teinture des sourcils et des dents longtemps en vigueur dans la haute aristocratie ${ }^{19}$.

Dans son allure : il délaisse les habits de Cour, sauf pour l'accomplissement des fonctions rituelles, pour le costume occidental dans ses fonctions civiles et l'uniforme en tant que commandant suprême des armées. Meiji apparaît à la Cour en frac brodé d'or à l'occidentale le 13 mai 1872 lors d'une audience accordée à un ancien noble de Cour, Hashimoto Saneakira (1809-1882), avant même qu'il ne passe à la coiffure occidentale. Mais on le voit pour la première fois en uniforme lors de la revue militaire qu'il préside quelques mois auparavant, le 2 mars $1872^{20}$. Dans ses appartements privés en revanche, il passe aux vêtements japonais. Il change ses habitudes alimentaires, moins pour des raisons de santé, que pour satisfaire aux règles du protocole diplomatique qui l'oblige désormais à accueillir des hôtes étrangers, et se met à boire du lait deux fois par jour en provenance des fermes impériales. Le premier repas à l'occidentale lui est servi le 9 février 1872, jour de l'an du calendrier lunaire.

19. Sous le Japon féodal, la coiffure et le type de chignon étaient des signes de distinction sociale. La classe des guerriers portait un style de coiffure distinctif, interdit aux gens du commun. Le changement de coiffure appartenait au registre des mesures visant à promouvoir la commune égalité des Japonais en tant que sujets de l'empereur. Sur l'évolution de l'apparence physique de l'empereur, NAKAMURA Ikuo, « Tôgyô, josô, dansô. Tennôzô no sanruigata », Les trois figures de l'empereur, enfant, féminisé et masculinisé in NAKAMURA Ikuo et TOGO Yoshio, Kokoro to kotoba ni tôzai no setten wo motomete, l'esprit et les mots : la rencontre entre l'Est et l'Ouest, Tôkyô, Hokushoku shuppan, 1990, p. 30 et s. ; YATSUKI Kimio, Tennô to nihon no kindai jô Kempô to arahitogami, La modernisation du Japon, vol.1 : Constitution et dieu incarné, Tôkyô, Kôdansha, 2001, p. 19-26. Memories, by Lord REDESDALE (Algernon Bertram Freeman-Mitford, Baron (1837-1916), New York, Dutton, 1915, vol. 2, p. 459-460, pour un point de vue occidental, à l'occasion de l'audience impériale accordée le 26 mars 1868 au ministre britannique Harry Smith Parkes (1828-1885).

20. MoriTA Toyoko, « Meiji tennô no yôsôka. Kunaichô shoryôbu shozô [goyô doroku] wo sankô ni », L'occidentalisation de l'empereur Meiji. A partir du registre des fournitures impériales conservé à la division des archives de l'agence de la Maison impériale, Nihon kaseigaku zasshi, 2015, vol. 66, nº p. 317-328. 
Trois semaines plus tard, le 3 mars, il goûte à la viande de bœuf. Cette initiative donne lieu à un incident : le 26 mars, pour protester contre cette violation des interdits alimentaires issus du bouddhisme, le Palais est envahi par dix adeptes de la secte religieuse Ongyô. L'incident se solde par quatre morts, un blessé grave et cinq arrestations. Par la suite, en juillet 1873, on lui fait apprendre les bonnes manières pour se tenir à table durant les banquets, ainsi que l'usage approprié des couverts ${ }^{21}$.

Enfin, les déplacements impériaux dans les provinces de l'empire, surtout durant la première moitié du règne, sont autant de manifestations de mise en scène, d'ostension et de monstration d'un corps impérial incarné, exposé au regard des foules japonaises ${ }^{22}$. Tous ces changements obéissent à des préoccupations politiques car il s'agit d'affirmer le nouveau visage de la monarchie japonaise, à l'intérieur comme à l'extérieur. Au sens propre, comme au sens figuré. La modernisation de l'apparence extérieure de l'empereur vise à intégrer le tennô, à parité, dans le cénacle des monarques occidentaux dont il emprunte les atours et la pose. Meiji portera la barbe, comme ses pairs européens. D'ailleurs, le port de la barbe, du sabre (occidental), la pratique de l'équitation pour passer les troupes en revue, l'uniforme, inscrivent le tennô dans une dimension supérieure de masculinité, alors que les codes traditionnels de représentation de la figure impériale empruntaient plus largement aux métaphores féminines et maternelles ${ }^{23}$. Là aussi, il s'agit de se démarquer de la posture impériale des premiers mois de la Restauration lorsque, lors des audiences accordées aux ministres étrangers au printemps 1868, le jeune Meiji apparaît sous des vêtements de Cour et un maquillage qui lui donnent, selon les observateurs occidentaux, une allure féminine. Le changement des habitudes alimentaires, comme par exemple la consommation de viande, jusque-là frappée par des interdits religieux, a valeur d'exemplarité dans le cadre d'une politique hygiéniste ayant pour but d'améliorer la condition physique de la population en général et des conscrits en particulier. L'ostension de l'empereur aux foules se démarque également du régime de quasi réclusion dans lequel était tenu le monarque dans son palais de Kyôto durant

21. MaJima Ayu, «Shoku niku to iu kindai. Meiji-ki ni okeru shoku niku gunji juyô to niku shokukan no tokuchô », Consommation de la viande et modernité. La demande militaire de viande à l'époque de Meiji et les conceptions relatives au régime carné, Kokusai kirisutokyô daigaku gakuhô 3-A Ajia bunka kenkyû bessatu, 2012, p. 213-215 ; MIYACHI Masato et YASUMARU Yoshio, Nihon kindai shisô taikei 5 Shûkyô to kokka, Traité sur la pensée du Japon moderne.Tome 5 : Religion et Etat, Tôkyô, Iwanami shoten, 1988 p. 175.

22. Yoshimi Shun.ya, «Les rituels politiques du Japon moderne : tournées impériales et stratégies du regard dans le Japon de Meiji », Annales, 1995, vol.50-2, p. 341-371. Egalement notre étude, « L'institution impériale en droit public japonais depuis la Restauration de Meiji », Thèse pour le doctorat d'Etat en droit, Université de droit d'économie et de sciences sociales de Paris (Paris 2), Paris, 3 février 1984, vol. 1, p. 92-95 ; HARA Takeshi, Kashisareta teikoku. Kindai nihon no gyôkôkei. L'empire révélé par l'image. Les tournées impériales en province dans le Japon contemporain, Tôkyô, Misuzu shobô, 2001.

23. Sur cet aspect, KANO Mikiyo, Josei to tennô, L'empereur et les femmes, Tôkyô, Shisô no kagakusha, 1979. 
toute l'époque d'Edo : il n'avait plus à être cette figure distante et abstraite, confisquée et cachée aux yeux des multitudes par la noblesse de Cour, entourée de superstitions selon lesquelles «il ne foulait jamais le sol de ses pieds $»^{24}$.

\section{Le corps impérial comme "corps précieux»}

Après la Restauration, la métamorphose physique de l'empereur, les transformations apportées à son mode de vie n'impliquent nullement une banalisation et une sécularisation du corps impérial et de ses représentations, mais plutôt une actualisation des interdits qui visent à préserver la distance symbolique et l'aura de sacralité qui sépare, radicalement, l'empereur de ses sujets, et distinguent le tennô de ses homologues étrangers. D'abord, la langue de Cour en vigueur au Palais continue à utiliser un terme particulier pour qualifier le corps de l'empereur, gyokutai, qui signifie littéralement «corps précieux », et lorsqu'il adresse à ses sujets par rescrits ou édits impériaux interposés, il n'utilise pas la langue vernaculaire, mais un idiome propre à la Maison impériale. Les rites de succession, et en particulier le Daijôsai, « transfigurent » le corps de l'empereur, comme investi par l'esprit de ses prédécesseurs $^{25}$. Ce « corps précieux » est juridiquement protégé : d'une part, la protection de l'intégrité physique des membres de la Famille impériale est assurée dès le premier code pénal promulgué en juillet 1880 . Le crime de lèse-majesté est repris aux articles 73 à 76 du nouveau code pénal du 24 avril 1907 : les attentats et tentatives d'attentats contre l'empereur, le prince héritier et son fils aîné, les trois impératrices ${ }^{26}$, sont passibles de la peine de mort (article 73$)^{27}$. Sur ce point, l'institution impériale japonaise ne se distingue guère des monarchies européennes où tout attentat contre la personne du prince justifie des peines plus sévères qu'un meurtre ou un assassinat ordinaire, voire des supplices spécifiques. D'autre part, l'article 3 du code de la Maison impériale dispose qu'en cas de «maladie grave et incurable de corps ou d'esprit ou d'obstacle sérieux », le conseil de la Maison impériale peut modifier l'ordre de succession au trône ». Le but de cette disposition est d'éviter que le trône

24. Voir à ce sujet le rapport d'Ôkubo Toshimichi (1830-1878), l'un des artisans de la Restauration en date du 16 février 1868, cité in YATSUKI K., op.cit., p. 21. Passage visiblement inspiré du Livre 2 de l'Histoire naturelle, civile et ecclésiastique du Japon d'Engelbert Kampfer (1651-1716), un médecin et naturaliste allemand qui avait séjourné au Japon entre 1690 et 1692, et qui avait été traduite à partir de sa version hollandaise en 1801 par Shizusa Tadao (1760-1806).

25. YAMAORI Tetsuo, Tennô no kyûchû saishi to nihonjin, L'empereur, les rites impériaux du Palais et les Japonais, Tôkyô, Nihon bungeisha, 2010, p. 37-40.

26. L’impératrice, l'impératrice douairière, l'impératrice archi-douairière.

27. Il en sera fait application en 1911 lorsque sera déjoué un projet d'attentat anarchiste contre le train de l'empereur. Le 24 janvier 1911, vingt-quatre condamnations à mort avaient été prononcées dont douze commuées. Parmi les condamnés exécutés, Kôtoku Shûsui, le principal idéologue de l'anarcho-syndicalisme japonais. De nombreux historiens doutent aujourd'hui de la participation de Kôtoku au complot contre la vie de l'empereur Meiji, même s'il ne s'est pas désolidarisé de ses co-accusés. Voir à ce propos Christine LEVY, "Kôtoku Shûsui et l'anarchisme », Ebisu Etudes japonaises, n²8, printemps-été 2002, p.61-86. 
n'échoie à un héritier qui serait, manifestement et durablement, hors capacité d'exercer les fonctions monarchiques. Elle n'a jamais été appliquée : la maladie dont a été atteint le prince impérial Yoshihito, dès sa naissance, n’a jamais été considérée comme un obstacle à la succession de son père en 1912.

Le respect $\mathrm{du}$ « corps impérial » en tant que corps public est encadré par des règles d'étiquette confinant aux tabous et interdits. Au passage d'un cortège impérial les fenêtres à l'étage doivent être fermées. Pour des raisons de sécurité, mais aussi parce qu'il est indécent de toiser l'empereur d'une position élevée. Lors des audiences, le protocole exige de ne pas lever les yeux sur lui et de s'adresser à lui en respectant les convenances linguistiques du langage de Cour. Lorsque le gouvernement va créer les premiers timbres modernes en avril 1871, puis uniformiser le système monétaire en créant le yen trois mois plus tard en juin 1871, il n'existera pas de pièces de monnaie, de billets de banque, de timbres à l'effigie de l'empereur ou du couple impérial, parce que leur détention et leur manipulation - le cachet de la poste par exemple - pourrait donner lieu à des actes sacrilèges. De même la confection, la diffusion, l'exposition des portraits impériaux feront l'objet, à partir de la seconde partie du règne, d'une réglementation minutieuse ${ }^{28}$. Il y a donc une volonté d'imposer une image contrôlée et stéréotypée du corps public de l'empereur, surtout depuis que les premières images photographiques officielles font apparaître le caractère gauche et emprunté d'un jeune prince, flottant dans son uniforme, avachi sur la chaise sur laquelle il pose, et manifestement mal à l'aise devant l'objectif ${ }^{29}$.

Selon la conception classique que l'époque de Meiji systématisera sous la forme extensive d'un « culte impérial » étendu à l'ensemble de la nation, l'empereur est « dieu manifeste ». Mais le corps de l'empereur est multiple : à titre individuel, il est périssable, assujetti aux afflictions, au déclin et aux maladies. Le corps politique du tennô repose, lui, sur trois éléments immuables : il est le dispensateur suprême de légitimité ; il s'insère dans une continuité dynastique unique et éternelle ; il a le monopole des rites et cérémonies qui lui ont assuré au cours de l'histoire la suprématie politico-religieuse sur les autres clans. Cette distinction n'implique pas cependant que ces deux corps de l'empereur soient radicalement séparés, car ils se rejoignent dans une dimension cosmique : la santé du monarque, sa longévité sont garantes de la prospérité, de l'harmonie sociale et de la paix. Mais une complexion faible, la maladie introduisent des facteurs d'instabilité, car elles sont le signe d'une malédiction envoyée par les dieux. Il convenait dès lors d'en identifier les causes et de

28. Sur ce point, notre étude : «Les portraits impériaux. Contribution à l'étude du culte impérial au Japon », Mélanges offerts à René Sieffert à l'occasion de son soixante-dixième anniversaire, Paris, 1994, Centre d'études japonaises, Institut National des Langues et Civilisations Orientales, p.437-450. Egalement, Mikiko HIRAYAMA, « The emperor's new clothes: japanese visuality and imperial portrait photography ». Journal History of Photography, 2009, vol. 33, n² 2, p. 165-184.

29. C'est la raison pour laquelle les photographies de l'empereur Meiji sont rares, prises à la dérobée et de loin. Il était de notoriété publique que l'empereur détestait être pris en photo. 
la conjurer par des rites divinatoires et propitiatoires appropriés, dans le but de purifier le corps de l'empereur et de restaurer par-là l'harmonie originelle. Le bouddhisme, surtout dans sa version ésotérique, fut également mis à contribution : prières, lectures de sutras, construction de temples et de statues bouddhiques participent également de toute une gamme de pratiques rituelles et religieuses, visant soit à protéger l'empereur de la maladie, soit à le guérir, soit à le protéger de la souillure $^{30}$. Quant aux interdits caractérisant le corps du souverain, ils ont fait l'objet, en Occident, de descriptions plus ou moins fantasmagoriques, fruits de ouï-dire et de légendes mal digérées, nourries par les mystères entourant confinement de l'empereur dans son palais de Kyôto ${ }^{31}$. Faut-il davantage se fier aux sources japonaises ? La principale source émane de Tsumura Sôan (1736-1806) qui, dans le Kantai, évoque la question, mais dans des termes passablement différents de Kampfer car il met l'accent sur les tabous que doivent respecter les dames d'honneur, en charge du service ordinaire de l'empereur, et qui renvoient à la prévention de la souillure dans le shintô. En bref, si les descriptions apparaissent plus « réalistes », leur authenticité n'est pas avérée car l'auteur ne cite pas ses sources. Depuis la fin du Moyen-âge japonais, selon l'historiographie dominante, on assiste bien à un renforcement des tabous concernant le corps impérial. Ainsi, les techniques médicales relevant de la médecine traditionnelle telles que la moxibustion ou l'acupuncture ont été prohibées à la Cour. Les pièces du Palais sont protégées lors des éclipses solaires et lunaires jugées néfastes pour le «corps précieux » de l'empereur ${ }^{32}$. Et il est intéressant d'observer que le renforcement de ces tabous intervient au moment même où le pouvoir politique de la Cour connaît un déclin prononcé. Comme si le besoin de sacraliser le corps impérial devait compenser, dans l'ordre symbolique, la diminution du pouvoir impérial dans la sphère politique. Le corps de l'empereur devient ainsi un lieu sanctuarisé au fur et à mesure même que le périmètre d'influence, géographique et politique, de la Cour se réduit. Il n'est est pas moins vrai que la nature et le mode opératoire de ces interdits sont encore aujourd'hui mal documentés. L'on sait seulement qu'ils faisaient peser une pression sur certains souverains qui ont tenté de s'en affranchir par l'abdication et qu'ils ont servi de prétexte à des règlements de compte politiques ${ }^{33}$.

30. NAKAMURA Hideo, Kodai saishi-ron, Considérations sur les rites impériaux à l'époque antique, Tôkyô, Yoshikawa Kôbunkan, 1999, en particulier le chapitre 3.

31. Outre les travaux précités d'Engelbert Kampfer qui s'inspiraient partiellement de lettres de Jésuites du XVI ${ }^{\mathrm{e}}$ siècle, il convient de mentionner le Puissant empire du Japon du français François Caron (1600-1674) qui avait vécu vingt ans au Japon et publié en 1648, ou le journal du Hollandais Conrad Cramer de 1626.

32. IHARA Kesao, Chûsei no kokka to tennô, girei, l'Etat au moyen-âge, l'empereur et les rites, Tôkyô, Kôkura shobô, 2012, p. 169 ; FuJITA Satoru, Tennô no rekishi 6 Edo jidai no tennô, Histoire de l'empereur vol. 6 l'empereur à l'époque d'Edo, vol.6, Tôkyô, Kôdansha, vol.6, p. 186-190 ; KURODA Hideo, Ô no shintai ô no shôzô, Corps royal et portraits royaux, Tôkyô, Heibonsha, 1993.

33. La renonciation à l'acupuncture date vraisemblablement de l'empereur Gohanazono (1419- 
L'introduction de la médecine occidentale au Palais fut certes un marqueur de modernité, mais elle n'entraîne pas la caducité de certaines des coutumes et usages qui touchent le traitement du corps impérial et avec lesquelles la médecine occidentale a dû nécessairement transiger. Traditionnellement, le corps de l'empereur est sacré et inviolable : il est le réceptacle de l'esprit de la déesse Amaterasu-Ômi-Kami, divinité fondatrice du clan impérial et, dans la tradition confucianiste, ce corps lui a été légué par ses ancêtres, et donc par les monarques qui l'ont précédé. En outre, ce corps impérial s'inscrit, du point de vue de l'anthropologie religieuse, dans la dialectique du pur et de l'impur - et des tabous qui y sont inhérents - qui rythment la vie du Palais. Les bains rituels qui précèdent les cérémonies shintô, comme les bains quotidiens, sont soumis à une étiquette rigoureuse car il n'est pas question que l'empereur se lave tout seul. Seules les dames d'honneur de rang supérieur ont la charge de la partie supérieure du corps, tandis que les dames d'honneur moins titrées ont la charge de la partie inférieure, et l'on veille à ce que cette eau-là ne soit pas utilisée pour laver la partie supérieure. Essuyer le corps impérial obéit également à la même dialectique et le yukata qu'enfile l'empereur après son bain ne doit pas comporter de coutures apparentes. Ce protocole eut-il raison de la patience de Meiji ? Toujours est-il que durant les dernières années de sa vie, l'empereur ne prend plus de bain que pendant l'été, et qu'il s'abstient de procéder de plus en plus en plus aux bains rituels, ce qui l'oblige à déléguer à des messagers impériaux ou à des hauts fonctionnaires du Palais le soin d'accomplir les rites à sa place ${ }^{34}$.

\section{LES PATHOLOGIES IMPERIALES A L'EPREUVE DE LA MEDECINE OCCIDENTALE}

Le règne de Meiji fut en partie paradoxal : il fut l'un des plus longs que l'archipel ait connus, mais l'empereur est mort, sinon précocement, du moins, à un âge relativement peu avancé. Surtout, la famille qu'il a fondée a été décimée par des deuils à répétition provoqués, dans la majorité des cas, par la même pathologie. L'empereur lui-même a été impliqué dans certaines des controverses médicales qui ont relancé le bras de fer entre médecine occidentale et médecine sino-japonaise et son rapport personnel à la médecine moderne est complexe. Enfin, au crépuscule du

1470) : on en trouve trace dans le Yasutomiki, les notes journalières d'un haut fonctionnaire de la Cour de Kyôto, Nakahara Yasutomi (1400-1457) pour la fin 1442. Il a été également soutenu que l'abdication de l'empereur Gomizunoo, en décembre 1629, aurait été motivée par l'impossibilité, en tant que souverain régnant, de se soigner correctement au moyen de la moxibustion. Mais il est plus vraisemblable qu'elle ait causée par les heurts à répétition entre le tennô et le bakufu. L'empereur Reigen (1654-1732) avait également écarté de la succession son propre fils aîné, le prince impérial Hitotsunomiya (1671-1701), au profit de son quatrième fils, le prince impérial Asahito (1675-1709), au motif qu'il avait eu recours à la moxibustion. En fait, l'empereur avait décidé de ne pas respecter l'accord conclu entre l'empereur retiré Gomizunoo, la Cour de Kyôto et le bakufu selon lequel la succession au trône échoirait au prince impérial Hitotsunomiya si l'épouse en titre de Reigen, ne lui donnait pas d'héritier.

34. Yonekubo Akemi, Meiji tennô no ichinichi. Kôshitsu shisutemu no dentô to genzai, Vie quotidienne de l'empereur Meiji. Le système de la Maison impériale à la lisière de la tradition et du contemporain, Tôkyô, Shinchôsha, 2006, p. 140-143. 
règne, l'agonie de l'empereur va illustrer à la fois les problèmes particuliers posés la maladie impériale et la médiatisation sans précédent dont elle a fait l'objet.

\section{Une mortalité infantile galopante et la controverse sur le béribéri}

L'empereur Meiji a eu quinze enfants dont dix sont morts en bas-âge, avant l'âge de deux ans. Seul un enfant mâle lui a survécu, le futur empereur Taïshô, handicapé lui-même par la méningite contractée peu de temps après sa naissance. Ce taux de mortalité effrayant est à lui seul une caricature de l'état sanitaire du Japon, où la mortalité infantile est importante, où le choléra, la tuberculose et le béribéri - ces deux dernières maladies qualifiées à juste titre de «fléaux nationaux », kokuminbyô -, font des ravages dans toutes les couches de la société. Il est vrai que l'endogamie séculaire qui oblige à ne choisir pour épouses impériales que des femmes issues de familles aristocratiques elles-mêmes apparentées à la Maison impériale, a pu conduire à aggraver les effets de la consanguinité. On a également incriminé le fard blanc en usage chez les femmes de la noblesse de Cour, dont la toxicité du fait de sa haute teneur en plomb et en mercure en cas d'allaitement n'a été révélée qu'en 1923, grâce aux travaux de Hirai Ikutarô (1865-1945), un pédiatre de l'Université de Kyôto. Enfin, on a souligné que les mariages précoces et l'absence de pédiatres attachés à la Famille impériale ont pu constituer des facteurs de risque supplémentaires. Quoi qu'il en soit, l'aristocratie japonaise dans son ensemble semble frappée par un mal endémique, la méningite, dont la prévalence au sein de la population japonaise était faible. Ce «mal aristocratique » fut responsable d'une grande partie des décès des nourrissons dans la noblesse japonaise et la Famille impériale ${ }^{35}$. Meiji avait été un père prolifique. En cela, il se conformait à une sinistre tradition : la succession des paternités était le seul antidote à la mortalité infantile dévorante à la Cour impériale. Aucun de ses enfants n'était né de l'impératrice, stérile, et les dames d'honneur étaient préposées au ventre, ce qui explique d'ailleurs que le « code de la Maison impériale » de 1889 n'excluait pas les enfants mâles naturels de la succession. La fragilité récurrente de la santé des enfants impériaux et de l'héritier au trône, outre qu'elle constituait un sujet de préoccupation personnel pour Meiji et sa descendance, ne pouvait que déstabiliser les services médicaux du Palais. Elle relança le débat sur la meilleure façon de soigner les membres de la Famille impériale, et plus particulièrement les enfants impériaux à naître. Fallait-il s'en tenir à une combinaison de soins mêlant médecine occidentale et médecine sino-japonaise ou uniformiser les protocoles, mais alors selon quelle ligne? Toujours est-il que Meiji prit toujours soin de pouvoir faire appel à des adeptes

35. FUKASE Yasuaki, « Meiji tennô kôshijo yôsetsu no shiin ni tsuite », A propos des causes de la mort prématurée des enfants de l'empereur Meiji, Nihon ishigaku zasshi, septembre 2015, vol. 61, n³, p. 255-266. Sur l'absence préjudiciable de pédiatres occidentalistes auprès de la Famille impériale, lettre de Hirota Chikaatsu à Ikeda Kensai datant du 8 mars (1887?), ibidem, p. 256-257. Egalement, Ottmar Von Mohl, Am Japanischen Hofe. Kammerherr Seiner Majestät des Kaisers und Königs Wirklicher Geheimer Legations-Rat, Berlin, Reimer, 1904, p. 211 et s. L'auteur avait été conseiller auprès du ministère de la Maison impériale entre 1887 et 1889 sur les questions protocolaires et cérémonielles. 
réputés de la médecine traditionnelle. Pourtant, l'Inspecteur général de la santé aux Armées, Hashimoto Tsunatsune, qui avait soigné la sixième fille de l'empereur, et après le décès de son quatrième fils le prince impérial Michihito (1887-1888) en novembre 1888 , n'avait pas manqué de souligner que puisque la médecine occidentale avait sensiblement amélioré le niveau santé de la population, l'excessive mortalité infantile dans la Maison impériale plaidait a contrario en faveur de ce type de médecine, d'une sélection plus rigoureuse des pédiatres et de l'abandon de la coutume visant à confier l'éducation des rejetons impériaux aux dames d'honneur de la Cour. En d'autres termes, la santé des enfants impériaux dépendait à la fois de l'amélioration de la qualité et de la spécialité des médecins, mais aussi d'une réforme des usages de la Cour, afin que les enfants impériaux en bas-âge soient pris en charge par des familles d'accueil en dehors du Palais, issues de l'ancienne classe des guerriers, et ayant l'expérience de ce type d'éducation ${ }^{36}$. Sur ce dernier point au moins, par l'entremise de l'impératrice, Hashimoto obtint satisfaction.

Si la médecine occidentale obtint progressivement droit de cité, il est cependant douteux que cette position dominante soit due à la conversion du prince aux vertus de la médecine occidentale. En 1878, alors que le béribéri fait rage à Tôkyô, dans les forces armées, et n'épargne pas le souverain à la fin mai, occidentalistes et traditionalistes s'affrontent sur l'origine de la maladie. Les occidentalistes soutiennent que le béribéri est une maladie endémique et que pour la soigner il faut changer d'environnement et privilégier les zones montagneuses. Voire qu'elle est d'origine bactérienne, selon la thèse développée par Bälz et son collègue de l'Université de Kyôto, Heinrich Botho Scheube (1853-1923) et longtemps retenue par l'Armée. Ils ont l'oreille du ministre de la Droite Iwakura Tomomi (1885-1883), l'un des hommes forts du régime. Mais Meiji écarte la climatothérapie, au motif qu'elle n'a pas sauvé la vie de l'une de ses filles, décédée le 2 septembre 1877, que le béribéri sévissant à l'état endémique dans l'archipel, il n'est pas possible d'obliger des pans entiers de la population à de tels déplacements, et que des militaires de l'Armée stationnés en zone montagneuse en ont été également victimes. Sur les conseils de deux de ses plus proches conseillers, Sasaki Takayuki (1830-1910) et Yoshii Tomozane (1828-1891), l'empereur appelle à la rescousse Tôda Chôan (1819-1889), un adepte de la médecine sino-japonaise de renom, spécialiste du béribéri, et qui avait été médecin auprès $\mathrm{du}$ $14^{\mathrm{e}}$ shôgun Tokugawa Iemochi (1846-1866), pour lequel cette maladie peut être soignée par une diversification des habitudes alimentaires - par exemple moins de riz blanc et plus de riz complet, de blé et de haricots - et la pharmacopée traditionnelle. Meiji en appelle à la coopération entre médecine chinoise, japonaise et occidentale et un hôpital spécialisé sur le béribéri est créé à cet effet en juillet 1878. Mais il n'est pas suivi par les occidentalistes de la direction des affaires médicales du ministère au motif que les solutions - pourtant efficaces - préconisées par Tôda, et qui commençaient à faire leurs preuves dans la Marine, n'étaient qu'un pâle décalque de

36. Meiji tennô-ki, op.cit., tome 6, p. 108-109, 509-510. 
la médecine occidentale, sans effet sur les malades les plus gravement touchés. En conséquence l'hôpital fut fermé en juin 1882. Pourtant les conclusions de Tôda furent confirmées par Takaki Kanehiro (1849-1920), un médecin de la Marine ayant étudié en Grande Bretagne, dans un rapport au trône en date du 29 novembre 1883 - alors que l'empereur souffrait d'une rechute depuis le mois d'août - qui soulignait que les pratiques alimentaires japonaises, pauvres en protéines, prédisposaient à la maladie. Auparavant, en octobre 1883, la direction des affaire médicales du ministère avait recommandé au ministre des Affaires Suprêmes que l'empereur et les enfants impériaux évitent les fortes chaleurs de l'été, propices selon elle aux rechutes, en séjournant dans des villas impériales construites à cet effet à $120 \mathrm{~km}$ de la capitale, à Nikkô et à Hakone, mais Meiji, pour ce qui le concerne, n'y donna pas suite ${ }^{37}$.

Cette controverse, qui divisa les milieux médicaux japonais, aura au moins deux conséquences : 1) faute de traitement approprié, l'empereur continuera à être victime du béribéri jusqu'en 1886 ;2) elle distillera auprès du souverain une méfiance à l'égard de la médecine occidentale, alors que celle-ci est en passe de supplanter la médecine traditionnelle à l'Université et à l'échelon national. D'autant plus que celle-ci est impuissante à enrayer les morts successives des enfants impériaux, ce qui amène Meiji à refuser à plusieurs reprises ultérieurement l'expertise médicale des médecins de la direction précitée et à lui préférer la médecine traditionnelle.

\section{Soigner l'empereur : un honneur...mais aussi des contraintes}

Soigner l'empereur, c'est d'abord avoir un accès privilégié au corps naturel du souverain, à son intimité, mais aussi aux espaces privés du Palais. Mais Meiji n'est pas un homme ordinaire, au moins à un triple point de vue. D'abord il est le chef de l'Etat et sa santé est de nature à affecter le fonctionnement du corps politique dont il est l'incarnation. Ensuite, ce corps naturel est investi d'une charge symbolique, émotionnelle et religieuse, particulière, car si la dimension cosmique de la santé impériale s'étiole après 1868 , le corps impérial demeure investi d'un caractère sacré. Enfin, il convient de tenir compte de la personnalité du monarque, de sa disponibilité et de sa capacité d'écoute. Autant de contraintes qui rendent la condition de médecin impérial particulièrement délicate.

37. Meiji tennô-ki, op. cit., tome 4, p. 399-400, 419, 637, 690 ; tome 6, p. 129-130 ; YAMASHITA Seizô, «Meiji-ki ni okeru kakke no rekishi wo meguru wadai», De quelques problèmes relatifs à l'histoire du béribéri au cours de la période de Meiji », Nihon ishigaku zasshi, juillet 1989, vol. 35, n³, p. 292-300 ; Alexander R. BAY, Beriberi in Modern Japan: The Making of a National Disease, University of Rochester Press, 2012, p. 30-33. SHINODA Tatsuaki, Rekidai tennô no karute, Le dossier médical des empereurs successifs, Tôkyô, Shinchôsa, 2006, p. 131-132. Sur Tôda, Christian OBERLANDER, «The rise of scientific medicine in Japan: beriberi as the driving force in the quest for specific causes and the introduction of bacteriology», Historia Scientarium: International Journal of the History of Science Society of Japan, mars 2004, vol.13, n4 4, p. 176-199 ; Donald KEEN, Emperor of Japan. Meiji and his World, 1852-1912, New York, Columbia University Press, p. 380-381. 
Globalement, il est difficile de se faire une idée de l'état de santé de l'empereur qui a suscité des appréciations contradictoires. Quatre constats cependant : il a grandi dans un environnement - la Cour impériale et ses dépendances - peu propice à la bonne santé des enfants impériaux ; les chroniques officielles font état de périodes de maladie fréquentes et parfois relativement longues, mais indiquent aussi que la pratique de l'équitation et des arts martiaux avaient fortifié son corps dans sa jeunesse ; il est en proie à des addictions : grand amateur de sake, mais aussi de vins de Bordeaux et de champagne, sa consommation excessive d'alcool fut vraisemblablement à l'origine du diabète qui l'a emporté en 1912 et il n'a jamais suivi les conseils de son entourage le mettant en garde contre ce travers. A partir de 1904, sa santé décline au plus fort de la guerre russo-japonaise et, en mai 1909, l'assassinat en gare de Harbin en Mandchourie de l'ancien Premier ministre et Résident général en Corée, Itô Hirobumi, ans, par un patriote coréen, An Jung-geung (1879-1910), fut un choc dont il ne s'est pas relevé. Itô n'avait pas été seulement son plus proche conseiller politique en trente ans, même s'il ne partageait pas le souhait de son ministre d'assouplir le système du «Cabinet transcendental ${ }^{38}$. Il avait été aussi un joyeux compagnon lors des soirées privées au Palais copieusement arrosées, et le seul à pouvoir se permettre en public des privautés qui auraient valu à tout autre une disgrâce sans appel.

A l'occasion, certaines de ces " indispositions 》 avaient même eu une tournure plus politique : en mars 1884, Itô Hirobumi avait cumulé ses fonctions gouvernementales d'Auditeur, avec la tête de la direction des enquêtes sur les institutions du Palais et du ministère de la Maison impériale. Ce cumul était destiné à aménager l'organisation et le fonctionnement du Palais dans la perspective de l'introduction prochaine du régime constitutionnel. Meiji en avait pris ombrage, craignant qu'Itô n'occidentalise jusqu'aux appartements privés du Palais. Non seulement l'empereur refusa de le recevoir pendant quelques temps, mais il bouda les affaires du gouvernement, n'accordant à Itô que deux heures quotidiennes dans la matinée. Itô avait même présenté sa démission à deux reprises, arguant que la maladie impériale n'était qu'un prétexte et que la mauvaise volonté de l'empereur était due à l'action pernicieuse du Grand chambellan Tokudaiji Sanetsune (1840-1919) et du lettré confucéen Motoda Nagazane (1818-1891), qui n'étaient pas au fait de la complexité des affaires de l'Etat et réputés pour leur conservatisme. La réconciliation entre les deux hommes n'interviendra qu'au printemps 1886 grâce aux efforts, notamment, de l'impératrice ${ }^{39}$.

38. Itô avait compris que le régime constitutionnel ne pouvait durablement fonctionner sans l'existence d'un parti majoritaire à la Diète, et en particulier à la chambre des Représentants et qu'en conséquence les membres du gouvernement ne pouvaient être choisis par le chef de l'Etat sans tenir compte de la composition du Parlement. Mais ce point de vue n'était pas partagé par les oligarques et l'empereur jugeait les partis politiques trop instables.

39. Sur cet épisode, Meiji tennô-ki, op.cit., tome 6, p. 339-342, p.446-449, 803-808. 
Dans sa vie quotidienne, Meiji est soumis à des contrôles de routine auxquels il se soumet difficilement : pouls, examen de la langue et des selles. Le soir à partir de de $21 \mathrm{~h}$, il a droit à une séance de massage. Mais l'empereur n'est pas un patient comme un autre. Les soins qui lui sont dispensés, mais aussi aux membres de la Famille impériale le sont à «domicile ». A l'époque, il n'existe pas encore d'hôpital dans l'enceinte du Palais. Durant la guerre sino-japonaise, en mars 1895, il y eut bien un moment le projet de transfert du Grand quartier général impérial installé à Hiroshima sur le continent pour superviser au plus près les opérations militaires, mais les préoccupations relatives à la santé impériale eurent raison de cette initiative. Il est vrai aussi que l'empereur avait tenu à partager l'inconfort du GQGI à Hiroshima pour soutenir le moral des troupes et donner l'exemple, alors que les armées japonaises devaient faire face aux rigueurs des hivers continentaux ${ }^{40}$. Les médecins impériaux étaient tenus de ne pas officier en blouse blanche. La blouse étant censée prémunir contre une éventuelle contamination en provenance du malade, se présenter devant l'empereur ou un membre de la Famille impériale dans cette tenue pouvait être considéré comme un acte d'incorrection, voire de lèse-majesté. A supposer même qu'un diagnostic scientifique ait été posé, indirectement sur la base des indications des chambellans et des dames de compagnie, directement à l'occasion des plaintes éventuelles du monarque lors des examens de routine, les médecins n'étaient pas toujours libres de prescrire les soins appropriés à l'état du patient : ceux-ci se limitaient principalement à la réforme des habitudes alimentaires et de l'hygiène de vie, et à l'examen des fluides. Ainsi, lorsque l'empereur fut diagnostiqué diabétique, les médecins recommandèrent de réduire sa consommation d'alcool et de sucreries. Encore que ces recommandations ne fussent pas transmises directement au tennô, mais seulement aux dames d'honneur en charge du service de la Table. L'empereur ne fut d'ailleurs jamais informé de la nature de sa maladie. Naturellement, le diagnostic ne fut pas rendu public pour ne pas affecter le moral des troupes sur le front lors de la guerre russo-japonaise. En tant que « corps précieux », il n'est pas non plus accessible aux techniques thérapeutiques invasives telles que les opérations, piqûres, saignées, purgations, perfusions, ligatures ou palpations jugées sacrilèges. Aucune indication officielle non plus sur le poids du monarque. De ce fait, lorsque l'état du malade justifie le recours à certaines de ces techniques, les médecins impériaux devaient parfois compter avec la frilosité des hauts fonctionnaires du Palais : le docteur Hirata Tsukasa (1859-1928) dut surmonter l'opposition des chambellans pour faire une piqûre de sérum au jeune Hirohito, le petit-fils de Meiji, atteint de diphtérie, sans autorisation impériale. La question du pouls a suscité nombre de discussions contradictoires. Selon les unes, la prise de pouls ne pouvait intervenir directement, mais par l'entremise d'une pièce de soie posée sur le poignet du monarque. Selon d'autres sources émanant du Palais, il s'agit de rumeurs infondées...

40. Rikugunshô-hen, ministère de l'Armée (éd.), Meiji gunji-shi. Meiji tennô go-denki shiryô, Histoire militaire de Meiji, documents biographiques relatifs à l'empereur Meiji, Tôkyô, Hara shobô, 1966, vol. 1, p. 992-993. 
Enfin, Meiji était un patient difficile. Pour les raisons évoquées plus haut, il n'aimait guère ni les médecins ni la médecine occidentale ${ }^{41}$. Il répugnait à être ausculté si bien qu'il avait fallu lui tailler des sous-vêtements ajourés spéciaux. Il a tendance à dissimuler la gravité des affections dont il est atteint : durant la guerre sino-japonaise, il qualifie de simple « refroidissement » ce qui est un commencement de pneumonie. Quand il prend froid, il se soigne à coup de décoctions de gingembre et de peau d'oranges. Si, de guerre lasse, il accepte de consulter un médecin, il se plaint alors des effets secondaires des médicaments. En février 1899, il décline la suggestion de ses médecins - relayée par le Grand chambellan Tokudaiji Sanetsune et le ministre de la Maison impériale Tanaka Mitsuaki (1843-1939) - de prendre du repos à Kyôto, l'ancienne capitale impériale. Et il s'irrite des articles de presse qui font état de sa corpulence ${ }^{42}$. Méfiance ? Mauvaise volonté ? Sans doute. Mais il y a aussi une part de stoïcisme de la part de Meiji, et un sens aigu du devoir : il ne veut pas que la maladie passe pour un aveu de faiblesse et s'il refuse de prendre du repos, c'est parce qu'il estime que les affaires de l'Etat passent avant son confort personnel.

L'un de ses proches, le chambellan, Hinonishi Sukehiro (1870-1942), confirme dans ses carnets que l'empereur était affaibli et fatigué en particulier depuis la mort d'Itô Hirobumi. Les rares clichés pris à la dérobée montrent une silhouette alourdie et il rentre épuisé des grandes manœuvres de l'Armée en novembre 1911. Les mixtions sont de plus en plus difficiles en raison de son insuffisance rénale et si, en public, l'empereur s'efforce de ne rien laisser paraître, en privé, il ne cache pas son désarroi : tantôt il souhaite en finir ; tantôt il s'inquiète pour l'impératrice cas de disparition ${ }^{43}$. L'une de ses favorites, la dame d'honneur, Yanagihara Naruko (1859-1943) - qui lui donna trois enfants dont le prince héritier Yoshihito - traduit le sentiment général : si Meiji vient à disparaître, la succession reviendra à son unique fils, dont la santé est elle-même déficiente. C'est donc la monarchie elle-même qui risque d'en être fragilisée. L'impératrice elle-même est amenée à le remplacer dans certaines de ses obligations protocolaires : audiences impériales, inaugurations, mise à l'eau des bâtiments de la Marine. Lorsqu'on lui conseille de prendre du repos et de consulter les médecins, le tennô s'emporte. Le docteur Bälz a beau recommander la construction de résidences de villégiature en dehors de la capitale et des cures thermales, jamais Meiji ne les fréquentera.

\section{L'agonie de l'empereur Meiji}

41. Lorsque la princesse impériale Chikako (1846-1877), ancienne épouse du $14^{\mathrm{e}}$ shôgun Tokugawa Iemochi décède du béribéri en septembre 1877, il faut deux heures pour que l'un de ses proches conseillers, Sasaki Takayuki (1830-1910), le convainque de se soumettre à un examen médical. D. Keene, op.cit., p. 291.

42. Meiji tennô-ki, op.cit., vol. 9, p. 595-596.

43. Meiji tennô no go-nichijô. Rinji teishitsu henshûkyoku ni okeru danwa sokki, Vie quotidienne de l'empereur Meiji. Notes de conversations prises à la direction provisoire des compilations historiques relatives à la Maison impériale, Kyôto, Shin gakusha kyôyûkan, 1976, p.55-58. 
La fin de Meiji est intéressante à cet égard, car, en dehors de la question de savoir si la médecine pouvait efficacement traiter l'empereur - ce qui était douteux compte-tenu de son état et des connaissances médicales de l'époque - elle concentre en l'espace de trois semaines les difficultés et contradictions auxquelles était confrontée la médecine impériale, en particulier dans la gestion des situations critiques.

30 juillet 1912, 0h43 : telle est la date officielle de la mort de l'empereur Meiji selon le ministère de la Maison impériale. Il n'avait pas encore soixante ans. En réalité, selon le témoignage d'un haut fonctionnaire du Palais, l'empereur rend le dernier soupir le 29 juillet à $22 \mathrm{~h} 43$, entouré des siens, après avoir murmuré à l'oreille de l'impératrice. Mais, pour des raisons liées à l'organisation des cérémonies d'accession de son fils, et compte tenu du délai nécessaire pour s'assurer du décès du souverain, la date officielle de la mort avait été retardée ${ }^{44}$. Le drame s'était noué trois semaines auparavant : le 11 juillet, le souverain avait participé à la cérémonie de remise des diplômes de l'Université de Tôkyô : il avait dû s'appuyer sur son épée pour marcher et sa démarche, dans les escaliers, était hésitante. De retour au Palais, il avait été pris de malaise. Le 14, l'empereur est victime de diarrhée et les médecins attachés au Palais en concluent à une gastroentérite, mais l'empereur, qui juge l'affaire bénigne, refuse les médicaments. Le 15, il assiste à une réunion du Conseil privé qui doit délibérer d'un accord russo-japonais sur la délimitation des zones d'influence en Mandchourie et de la normalisation des relations russo-japonaises. Il s'assoupit à trois reprises et le président du Conseil privé, Yamagata Aritomo (1838-1922), doit taper le sol de son sabre pour le réveiller. Il continue néanmoins à vaquer vaille que vaille à ses obligations gouvernementales. Le 19, alors qu'il fait $34^{\circ}$ à Tôkyô, et qu'il dîne, il se lève brusquement de table en se plaignant de troubles de la vue et s'écroule en allant aux toilettes. Le 20, les hautes instances du Palais hésitent alors sur la marche à suivre. L'impératrice donne son accord pour faire appel à deux sommités de la médecine occidentale de l'Université de Tôkyô. Cet ultime épisode suggère que la médecine palatiale était dépassée ou redoutait de prendre des initiatives susceptibles d'être désavouées. En conséquence, les professeurs Aoyama Tanemichi (1859-1917) et Miura Kinnosuke (1864-1950) - un ancien assistant de Bälz en 1887 sont appelés d'urgence au chevet de l'empereur. Yamagata, de son côté, souhaite que les techniques médicales les plus récentes soient utilisées. Les deux médecins confirment le diagnostic d'urémie établi la veille par le médecin chef du Palais Oka Genkei. Compte-tenu de l'urgence, on décide néanmoins de s'affranchir partiellement du protocole : les deux médecins, qui se relaient, peuvent se présenter en redingote. On leur épargne de se coiffer de l'eboshi traditionnel et de revêtir la tenue dite hitatare, mais, par respect, ils ne peuvent approcher l'empereur qu'en marchant sur les genoux, shikkô, Et quand Miura veut vérifier le réflexe ostéotendineux, on lui objecte

44. Cet article n'aborde pas la question des obsèques impériales. Voir à ce sujet, François MACE, « Les funérailles des souverains japonais », Cahiers d'Extrême-Asie, 1988/4, p. 157-165. 
qu'il est indécent que l'empereur montre son genou. Il ne peut pas non plus poser le stéthoscope sur le dos de l'empereur car un général, qu'il qualifia plus tard de « mentalement dérangé », s'y oppose ! Le 28, Meiji est pris de convulsions. Il est mis sous assistance respiratoire - une technique nouvelle à l'époque et qui, curieusement, ne fit pas difficulté - et, sur autorisation de l'impératrice et du prince héritier, on lui administre $\mathrm{du}$ sérum physiologique et du camphre pour ses propriétés anti-spasmodiques $^{45}$. Le 20 juillet, à $10 \mathrm{~h} 30$, le Journal Officiel annonce l'« indisposition de Sa Majesté », gofurei, selon la formule consacrée. Les informations diffusées rappellent que l'empereur était atteint de diabète depuis la fin 1904, puis à partir de janvier 1906, de déficience rénale chronique. A partir du 15, une gastroentérite s'était déclarée ; dans la nuit du 19 au 20, la fièvre était brusquement montée à $40,5^{\circ}$; le malade était tombé dans un demi-coma ; le rythme cardiaque était monté à 104 pulsations par minute et la fréquence respiratoire à 38 cycles par minute...

A l'annonce de ce communiqué, l'opinion est stupéfaite : c'est la première fois qu'elle est informée de l'état de santé du tennô. Les médecins impériaux, blâmés en interne pour n'avoir pas su soigner efficacement l'empereur depuis 1904, répondent que Meiji a refusé leurs services et qu'ils n'ont pas osé passer outre. La presse va bientôt leur reprocher leur silence, voire leur incompétence. Elle s'étonne de l'absence d'infirmières qualifiées au chevet du mourant. Et si l'empereur est réticent à se faire soigner depuis des années pourquoi les hauts dignitaires du régime et les membres de la Famille impériale ne sont-ils pas intervenus pour tenter de le fléchir ? En bref, sans l'exiger formellement, on s'attend à ce que les médecins impériaux démissionnent en bloc $^{46}$. Dans la capitale, on peine à prendre la mesure des événements : les vendeurs à la criée des numéros spéciaux des journaux se font même un moment rabrouer par la police qui suspecte une opération bassement commerciale $^{47}$. Une vague d'émotion submerge néanmoins rapidement le pays et le Nijû bashi, l'une des principales voies d'accès au Palais impérial, est bientôt envahi par la foule qui se prosterne et prie pour le rétablissement du monarque : un spectacle qui ne laisse pas d'étonner les étrangers sur place ${ }^{48}$. Une mobilisation spontanée et sans précédent, mais très vite relayée par les journaux, et la police doit intervenir pour

45. HAYASHI Eiko, Kindai igaku no senkusha. Miura Kinnosuke Meiji tennô Taishô tennô no o-isha san. Un précurseur de la médecine moderne : Miura Kinnosuke, médecin au service des empereurs Meiji et Taishô, Tôkyô, Sôbunsha, 2011, p.166-176.

46. Meiji tennô-ki, op.cit., vol. 11, p. 813 ; Bôjô Toshinaga, Kyûchû gojûnen, Cinquante ans au Palais, Tôkyô, Meitoku shuppansha, 1960, p. 23 ; Tôkyô Asahi shinbun, 22-23 juillet 1912, 5 août 1912 ; Pour certains auteurs, cette charge contre les médecins impériaux pourrait s'expliquer par un règlement de compte à l'intérieur du milieu médical contre la position dominante occupée par les facultés de médecine des universités impériales : SHINODA T, op.cit., p. 133 et s.

47. Tôkyô Asahi shinbun,, 21 juillet 1912.

48. Ibidem, 27 juillet 1912 ; Jiji shinpô, 25 juillet 1912. 
en encadrer le flux. A partir du 21, la Bourse de Tôkyô dévisse, et la presse se fait largement l'écho des informations officielles concernant le pouls, la respiration, les selles, l'urine, l'appétit du monarque. Le parallèle avec le dispositif d'information officielle mis en place au moment de l'agonie de son petit-fils, l'empereur Shôwa (1901-1989), au cours de l'hiver 1988-1989 est ici frappant. Le ministère de l'Intérieur indiqua aux préfets que l'« indisposition de l'empereur » ne requérait aucune mesure administrative spéciale, mais les théâtres, quartiers et lieux de plaisirs; réduisirent ou suspendirent leurs activités et les municipalités encouragèrent leurs administrés à faire preuve de «retenue ${ }^{49}$. Dans tout le pays, les sanctuaires shintô et les temples bouddhistes organisent des prières pour le rétablissement de l'empereur et les écoles sont également mobilisées à cet effet ${ }^{50}$. Les églises chrétiennes, toutes tendances confondues, ne sont pas en reste et des services particuliers pour la guérison du tennô sont célébrés dans les établissements de culte. Le Palais dépêche quant à lui des messagers impériaux auprès du Grand sanctuaire d'Ise voué au culte de la déesse solaire Amaterasu-Ômi-Kami, la divinité tutélaire de la Famille impériale.

La ferveur populaire engendra également quelques débordements. Il y eut dans la capitale, des propositions pour arrêter le trafic des tramways qui circulaient déjà au ralenti aux abords du Palais pour ne pas indisposer par leur bruit le repos de l'illustre patient. On exigea des officiels se rendant au Palais pour y présenter leurs devoirs ou s'informer qu'ils laissent attelages et voitures à distance du Palais. Certains, connaissant l'aversion du souverain pour l'éclairage électrique, préconisèrent le retour, au moins provisoire, aux chandelles et aux lanternes...Peu de voix s'élèveront contre ces excès. L'écrivain Natsume Sôseki (1867-1916), mais seulement dans son journal intime en date du 20 juillet, consigne qu'ils redoute que l'émotion populaire, légitime, soit prétexte à des démonstrations contestables d'autorité, et que la pression sociale en faveur d'une « retenue » excessive - et pilotée par le haut - ne suscite des frustrations dans la population qui pourraient se retourner contre la Maison impériale. On ne trouvera plus de trace de la mention de la maladie de l'empereur dans son journal jusqu'à l'annonce de son décès... ${ }^{51}$

La santé du monarque, comme celle de tout chef d'Etat, était naturellement un sujet majeur de préoccupation. Non seulement parce qu'elle était cruciale dans un système politique axé sur la figure impériale, mais aussi, et peut-être surtout, parce

49. Bôchô shinbun, 23 juillet 1912.

50. Jiji shinpô, 22 juillet 1912 ; The New York Times, 22 et 29 juillet 1912 ; The Milwaukee Sentinel, 22 juillet 1912. 398.

51. Sôseki zenshû, Oeuvres complètes de Sôseki, Tôkyô, Iwanami shoten, 1996-1997, tome 20, p. 
que cette problématique se situe sur la ligne de crête entre le corps politique du tennô, investi d'une dimension transcendante et son corps naturel pris dans sa dimension contingente. Toutefois le corps impérial ne s'inscrit pas dans une construction binaire et contrastive, mais dans une logique symbiotique qui n'est pas exempte de tensions entre le corps privé de l'empereur, dissimulé, son corps public, exposé, et son corps politique comme être de souveraineté. Dans ce cas, la préservation du corps politique du tennô comme être de souveraineté est prioritaire, non sans ambiguïté. C'est ce qui est arrivé le 25 novembre 1921, lorsque Hirohito assuma les fonctions de régent pour le compte de son père malade. Mais ce dernier restait néanmoins tennô. La régence maintenait intacte la fiction du corps politique de l'empereur, tout en autorisant le prince héritier à en exercer les fonctions gouvernementales. Encore que cette subrogation ne fût pas totale puisque le régent n'était pas autorisé à exercer les prérogatives impériales rituelles, tandis qu'en tant qu'individu, le corps privé de l'empereur était occulté et rejeté en dehors de l'espace public. Le cas de Meiji était différent puisque sa maladie s'inscrivait dans un processus normalisé et conventionnel. Soit il se rétablissait, et il reprenait normalement le cours de ses activités. Soit sa santé allait continuer à décliner, et l'on entrait dans un processus balisé de dévolution, puisque seul le décès de l'empereur ouvrait la succession impériale. En juillet 1912, il apparaissait cependant clair que la maladie allait sceller, à terme, le sort du monarque, et que la phase de transition politique, et donc le transfert du corps politique sur l'héritier présomptif, allait s'ouvrir, même si juridiquement, le gouvernement ne pouvait se permettre d'anticiper la succession et doit se contenter de gérer la situation créée par la maladie impériale ${ }^{52}$. A aucun moment l'hypothèse d'une régence n'a été envisagée car, une fois le tennô entré en agonie, la mort apparaissait comme la seule issue possible. Le communiqué du 20 juillet et les autres qui suivirent n'avaient pas pour objet de rassurer la population, mais bien de la préparer à l'inévitable. Hélas, après la disparition de Meiji, le répit sera de courte durée. Le nouveau règne allait très rapidement reposer la question de la santé - physique et mentale cette fois-ci - du nouvel empereur. Et il faudra attendre le 22 septembre 1988, pour que, pour la première fois dans l'histoire de la monarchie japonaise, le bistouri s'attaque au « corps précieux » du monarque. Hirohito avait 87 ans.

ERIC SEIZELET

CRCAO (Centre de Recherche sur les Civilisations de l'Asie Orientale)

52, rue du Cardinal Lemoine, 75005 Paris

eseizelet1@gmail.com

52. Le 26 juillet 1912, un conseil de Cabinet décide qu'en cas d'urgence, et sur autorisation du Premier ministre, les ministres responsables sont autorisés à prendre seuls les décisions relevant de leur ressort. Celles-ci feront l'objet d'un rapport au trône pour sanction, une fois le rétablissement de l'empereur, avec validité rétroactive : JACAR, code de référence A13100009800, https://www.digital.archives.go.jp/das/image/M0000000000001748415. Site consulté en avril 2018. 


\section{Résumé/Abstract}

\section{Eric SEIZELET}

Corps impérial, corps souffrant au Japon.

L’empereur Meiji et la médecine occidentale

Dans cet article, il s'agit moins de s'interroger sur conséquences des maladies de l'empereur Meiji sur le fonctionnement des institutions japonaises que d'analyser les problèmes posés par la gestion du corps impérial dans un contexte de modernisation accélérée, caractérisé par le passage de la médecine sino-japonaise à la médecine occidentale. A travers la question de la santé de l'empereur Meiji et de l'évolution des structures palatiales spécialisées, il analyse les interférences entre le corps privé de l'empereur, caché, son corps public, officiel, et son corps politique comme être de souveraineté. Il en conclut que dans le cas du Japon, la préservation de cette troisième dimension est capitale à la monarchie japonaise dont la légitimité s'inscrit davantage dans la continuité et l'unicité dynastiques que dans les figures éphémères et transitoires des monarques occupant le trône.

MoTS-CLES : Japon, empereur Meiji, santé, médecine, modernisation, corps impérial, vie politique

\section{Eric SEIZELET}

Imperial Body, Suffering Body in Japan.

Emperor Meiji and Western Medicine

The purpose of this article is not the description of the consequences of Meiji emperor's illness on the functioning of the Japanese political institutions, but the analysis of the various problems concerning the manipulation of the imperial body, in the wake of a rapid modernization process characterized by the shift from traditional to western medicine. Through the Meiji emperor health issue and the evolution of the specialized Palace offices, it studies the intertwinned relationships between the private - hidden - body of the emperor, his public - exposed - body, and its political body as locus of sovereignty. As a conclusion, it underlines the superiority of this last dimension for the preservation of the monarchy, the legitimacy of which is rooted in the continuity and unicity of the dynasty rather than in any individual and transitory figures of rulers on the throne.

KEYWORDS: Japan, Meiji emperor, health, medicine, modernization, imperial body, politics 